\title{
EXPERIMENTAL STUDY OF INTERMITTENT CROSSCLAMPING WITH FIBRILLATION AND MYOCARDIAL PROTECTION: REDUCED INJURY FROM SHORTER CUMULATIVE ISCHEMIA OR INTRINSIC PROTECTIVE EFFECT?
}

Ryuzo Bessho, MD*

David J. Chambers, PhD
Objective: During coronary artery revascularization, some surgeons favor intermittent crossclamping with ventricular fibrillation in preference to cardioplegic ischemic arrest for myocardial protection. It is unclear, however, whether intermittent crossclamping with fibrillation is equally protective or whether ischemic injury is reduced as a consequence of shorter cumulative ischemia.

Methods: We used isolated, Langendorff-perfused rat hearts, measured preischemic function (left ventricular developed pressure) with an intraventricular balloon, and then subjected the hearts to either (1) 40 minutes of global ischemia, (2) a 2-minute infusion of cardioplegic solution and 40 minutes of ischemia, (3) multidose (every 10 minutes) infusions of cardioplegic solution during 40 minutes of ischemia, (4) continuous ventricular fibrillation during 40 minutes of ischemia, $(5)$ intermittent $(4 \times 10$ minutes $)$ ischemia with 10 minutes of reperfusion, $(6)$ intermittent $(4 \times 10$ minutes $)$ ischemia preceded by intermittent cardioplegia, $(7)$ intermittent $(4 \times 10$ minutes $)$ ischemia with ventricular fibrillation, (8) continuous (40 minutes) ventricular fibrillation during coronary perfusion, or $(9)$ intermittent $(4 \times 10$ minutes $)$ ventricular fibrillation (with perfusion). All protocols were followed by 60 minutes of reperfusion.

Results: After 60 minutes of reperfusion, the percentage recovery of left ventricular developed pressure for groups 1 through 9 was as follows: $32 \% \pm$ $2 \%, 57 \% \pm 6 \%, 82 \% \pm 3 \%, 19 \% \pm 3 \%, 73 \% \pm 3 \%, 70 \% \pm 3 \%, 78 \% \pm 4 \%$, $55 \% \pm 2 \%$, and $57 \% \pm 3 \%$, respectively. No significant differences were identified among groups 3,5 , and 7 , but the percentage recovery of developed pressure in group 3 was significantly higher than that in group 6; the degree of recovery in groups 3 and 5 to 7 was significantly $(P<.05)$ higher than in groups $1,2,4,8$, and 9 . Early recovery was significantly $(P<.05)$ more rapid in groups 3 and 5 to 9, reaching a plateau (of 55\%-80\%) by 10 minutes of reperfusion; in groups 1,2 , and 4 , the recovery plateau occurred after 50 minutes. Left ventricular end-diastolic pressure was elevated in groups 1,2, and 4 but was almost unchanged from baseline in the other groups.

Conclusions: A similar level of myocardial protection was achieved with multidose (intermittent) cardioplegia or intermittent crossclamping (with or without fibrillation), indicating that intrinsic preservation by intermittent crossclamping with fibrillation does not exacerbate ischemic injury. (J Thorac Cardiovasc Surg 2000;120:528-37)
From Cardiac Surgical Research/Cardiothoracic Surgery, The Rayne Institute, Guy's and St Thomas' Hospital NHS Trust, St Thomas' Hospital, London, United Kingdom.

Received for publication Jan 11, 2000; revisions requested Feb 8, 2000; revisions received April 18, 2000; accepted for publication May 18, 2000.

Address for reprints: David J. Chambers, PhD, Cardiac Surgical Research/Cardiothoracic Surgery, The Rayne Institute, Guy's and St Thomas' Hospital NHS Trust, St Thomas' Hospital,
London SE1 7EH, United Kingdom (E-mail: david.chambers@kcl.ac.uk).

${ }^{*}$ Visiting research fellow from the Division of Cardiovascular Surgery, the Department of Surgery II, Nippon Medical School, Tokyo, Japan.

Copyright $\odot 2000$ by The American Association for Thoracic Surgery

0022-5223/2000 $\$ 12.00+0 \quad \mathbf{1 2 / 1 / 1 0 8 6 9 3}$

doi: $10.1067 / \mathrm{mtc} .2000 .108693$ 
D uring cardiac surgery, cardioplegic ischemic arrest is the preferred method for myocardial protection. ${ }^{1,2}$ However, for isolated myocardial revascularization (coronary artery bypass surgery), the technique of intermittent ventricular fibrillation (VF), either with continued coronary perfusion ${ }^{3}$ or, more commonly, with aortic crossclamping, ${ }^{1,4}$ has been favored by a minority of surgeons.

Recently, the use of intermittent crossclamping with VF for myocardial protection has been re-evaluated because of its simplicity and because of interest in a potential connection between intermittent crossclamping with VF and myocardial protection induced by ischemic preconditioning. Randomized clinical trials have shown that myocardial preservation by means of intermittent crossclamping with VF is equal to or better than that obtained with cold cardioplegia, ${ }^{5-11}$ assessed by clinical outcome, $, 8,11$ cardiac specific markers of injury (such as creatine kinase MB isoform or troponin $\mathrm{T}$ assays), ${ }^{6,7,11}$ free radical activity (such as lipid peroxidation and plasma antioxidant status), ${ }^{9}$ and from preoperative and postoperative electrocardiographic changes. $6,9,11$ Invariably, the cumulative ischemic period for intermittent crossclamping with VF during coronary surgery was relatively short (30-40 minutes) and was generally significantly shorter than the ischemic period when cardioplegic protection was used. ${ }^{5,711}$ Thus, from these clinical studies, it remains unclear whether intermittent crossclamping with VF is associated with an intrinsic myocardial protective effect or whether the shorter ischemic duration results in less severe myocardial injury.

We therefore conducted an experimental study to investigate whether intermittent crossclamping with VF has intrinsic myocardial protection and to compare any protective effect of this technique with those of other methods of elective cardiac arrest used during cardiac surgery procedures.

\section{Material and methods}

Animals. Adult male Wistar rats (240-300 g body weight) were used (Bantin and Kingman, Hull, United Kingdom). All animals received humane care in accordance with the "Guidance on the Operation of the Animals (Scientific Procedure) Act of 1986" published by Her Majesty's Stationery Office, London, United Kingdom. Rats were anesthetized with $95 \%$ oxygen and $5 \%$ carbon dioxide bubbled through diethyl ether; they were then anticoagulated with heparin (1000 IU/kg body weight) administered intravenously.

Heart isolation and perfusion. Hearts were rapidly excised from the anesthetized rat and immersed in cold $\left(4^{\circ} \mathrm{C}\right)$ Krebs-Henseleit buffer. The aorta was then cannulated and the heart perfused in the Langendorff mode with Krebs-
Henseleit buffer at a constant pressure $(75 \mathrm{~mm} \mathrm{Hg})$ and temperature $\left(37^{\circ} \mathrm{C}\right)$ within 30 seconds of excision. The pulmonary artery was incised to allow free drainage of coronary effluent.

An ultrathin intraventricular balloon, constructed from cling film (Saran Wrap; S. C. Johnson, Racine, Wis) over a 20-gauge cannula and made to match the internal dimensions of the left ventricle, was then introduced through the mitral valve into the left ventricle. The balloon, attached to a pressure transducer that in turn was connected to a chart recorder system (Gould Instruments, Oxnard, Calif), was gradually inflated with water to give a stable left ventricular end-diastolic pressure (LVEDP) of 3.0 to $8.0 \mathrm{~mm} \mathrm{Hg}$. This isovolumic state was maintained throughout the protocol.

All hearts were subjected to an equilibration period of aerobic perfusion for 20 minutes, and baseline readings of left ventricular systolic pressure (millimeters of mercury), LVEDP (millimeters of mercury), heart rate (beats per minute), and coronary flow rate (milliliters per minute) were then taken. Left ventricular developed pressure (LVDP) was calculated as the left ventricular systolic pressure minus LVEDP. Coronary flow rate was measured by timed collections of the coronary effluent. The development of contracture during ischemia was recorded by means of the intraventricular balloon.

Exclusion criteria. Hearts not satisfying preassigned exclusion criteria at the time of the baseline readings (after 20 minutes of aerobic perfusion) were excluded from the study. The acceptable ranges for LVDP, heart rate, and coronary flow rate were more than $100 \mathrm{~mm} \mathrm{Hg}, 220$ beats/min, and 8 to $16 \mathrm{~mL} / \mathrm{min}$, respectively.

Perfusion medium. The perfusion medium was a modified Krebs-Henseleit bicarbonate buffer with the following composition (in millimoles per liter): $\mathrm{NaCl}, 118.5 ; \mathrm{NaHCO}_{3}$, 25.0; $\mathrm{KCl}, 4.8 ; \mathrm{MgSO}_{4}, 1.2 ; \mathrm{CaCl}_{2}, 1.4$; and glucose, 11.0. The buffer was prepared daily, filtered through a $5-\mu \mathrm{m}$ poresize cellulose nitrate membrane filter before use, and continuously gassed with a mixture of $95 \%$ oxygen and $5 \%$ carbon dioxide to give a $\mathrm{pH}$ of 7.4 at $37^{\circ} \mathrm{C}$.

Preparation and administration of cardioplegic solution. In studies involving cardioplegia, the St Thomas' Hospital cardioplegic solution No. 2 (STH2), with the following composition (in millimoles per liter), was used: $\mathrm{NaCl}$, 110.0; $\mathrm{MgCl}_{2} \cdot 6 \mathrm{H}_{2} \mathrm{O}, 16.0 ; \mathrm{KCl}, 16.0 ; \mathrm{CaCl}_{2} \cdot 2 \mathrm{H}_{2} \mathrm{O}, 1.2$; and $\mathrm{NaHCO}_{3}, 10.0$. STH2 was prepared daily; $\mathrm{pH}$ was adjusted to 7.8 at $37^{\circ} \mathrm{C}$ and then filtered through a $5-\mu \mathrm{m}$ filter before use. The solution was delivered at $37^{\circ} \mathrm{C}$ and at a pressure of $45 \mathrm{~mm} \mathrm{Hg}$ for 2 minutes every infusion.

Induction and termination of VF. VF was induced by an electrical fibrillator (model G570, Department of Bioengineering, St Thomas' Hospital) by passing alternating current through two electrodes. The silver electrodes were coated with silicone; one was attached to the apex of the ventricle and the other to the aortic cannula for grounding. The minimum voltage necessary to achieve an alternating current that maintained VF was used. 


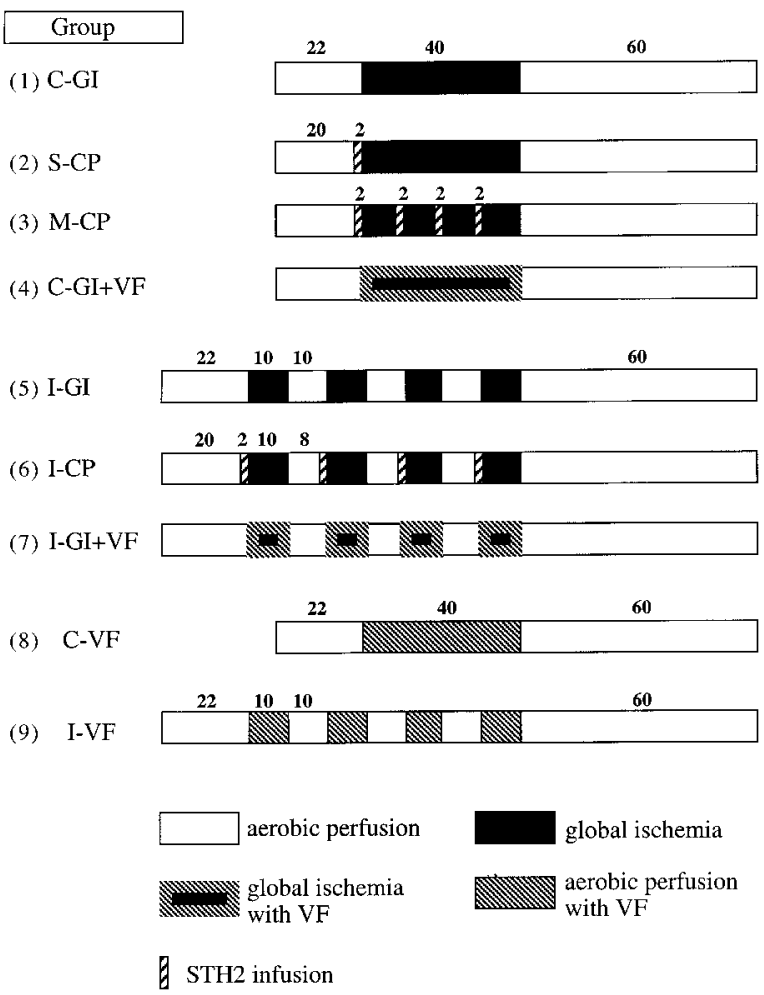

Fig 1. Experimental perfusion protocols. Nine groups of hearts $(\mathrm{n}=$ at least 6 per group) were studied: group 1: 40 minutes of continuous global ischemia $(C-G I ; \mathrm{n}=9)$; group 2: a single-dose infusion of St Thomas' Hospital solution 2 (STH2) and 40 minutes of global ischemia $(S-C P ; \mathrm{n}=9)$; group 3: multidose infusions of STH2 (every 10 minutes) throughout 40 minutes of global ischemia $(M-C P ; \mathrm{n}=6)$; group 4: continuous VF throughout 40 minutes of global ischemia $(C-G I+V F ; \mathrm{n}=6)$; group 5: $4 \times 10$ minutes of global ischemia (with 10 minutes of reperfusion) $(I-G I ; \mathrm{n}=6)$; group 6: $4 \times 10$ minutes of global ischemia with intermittent $\mathrm{STH} 2$ (with 10 minutes of reperfusion) $(I-C P ; \mathrm{n}=6)$; group 7: $4 \times 10$ minutes of global ischemia with intermittent VF (and 10 minutes of reperfusion) $(I-G I+V F ; \mathrm{n}=6)$; group 8: 40 minutes of continuous $\mathrm{VF}$ with coronary perfusion $(C-V F$; $\mathrm{n}=6$ ); group 9: $4 \times 10$ minutes of VF with coronary perfusion $(I-V F ; \mathrm{n}=6)$. All protocols were followed by 60 minutes of reperfusion.

If VF occurred during reperfusion or did not terminate spontaneously after the fibrillator was turned off, it was terminated by the use of a defibrillator (model G434, Department of Bioengineering, St Thomas' Hospital).

Experimental design. The perfusion protocols are shown in Fig 1. After equilibration, hearts were randomly assigned to 1 of 9 groups. All hearts ( $n=$ at least 6 per group) were subjected to one of the following protocols: (1) continuous global ischemia for 40 minutes (C-GI; $n=9$ ); (2) a single-dose infusion of STH2 before 40 minutes of continuous global ischemia (S-CP; $n=9)$; (3) multidose infusion of STH2 (before and every 10 minutes) throughout 40 minutes of continuous global ischemia (M-CP; $n=6)$; (4) 40 minutes of continuous global ischemia with electrically induced $\mathrm{VF}$ throughout (C-GI+VF; $n=6$ ); (5) 4 intermittent episodes of 10 minutes of global ischemia and 10 minutes of reperfusion (I-GI; $\mathrm{n}=6$ ); (6) 4 intermittent episodes of 10 minutes of global ischemia preceded by 2 -minute infusions of STH2 and followed by 8 minutes of reperfusion (I-CP; $n=6$ ); (7) 4 intermittent episodes of 10 minutes of global ischemia with electrically induced VF followed by 10 minutes of reperfusion in sinus rhythm (I-GI+VF; $n=6$ ); (8) continuous electrically induced VF for 40 minutes with coronary perfusion $(\mathrm{C}-\mathrm{VF} ; \mathrm{n}=6)$; and (9) 4 intermittent episodes of 10 minutes of electrically induced VF with coronary perfusion followed by 10 minutes of coronary perfusion in sinus rhythm (I-VF; $n=6$ ). LVEDP (intracavity pressure) was measured throughout these protocols.

All protocols were followed by a further 60 minutes of reperfusion, when recovery of myocardial function (LVDP, LVEDP, coronary flow rate, and heart rate) was measured.

Expression of results. Postischemic recovery of LVDP was expressed as a percentage of baseline values at the end of 20 minutes of aerobic perfusion; LVEDP was expressed as absolute values (millimeters of mercury). The following measurements related to contracture during ischemia were also noted: (1) time to onset of contracture (minutes), (2) time to peak contracture (minutes), and (3) magnitude of peak contracture (millimeters of mercury).

Statistics. Statistical analysis was performed with StatView and SuperANOVA software (Abacus Concepts, Inc, Berkeley, Calif) on an Apple Macintosh computer (Apple Computer, Cupertino, Calif).

All data are reported as mean \pm SEM. Comparisons between groups were assessed for significance by 1-way analysis of variance with post hoc analysis by means of the Fisher test, which allowed for multiple comparisons. A value of $P<.05$ (probability of $<5 \%$ that a difference between groups occurred by chance) was considered statistically significant.

\section{Results}

Measurement of baseline values of cardiac function. The mean baseline values for LVDP, coronary flow rate, heart rate, and LVEDP at the end of 20 minutes of aerobic perfusion are shown in Table I; there were no significant differences in any of these values between groups.

Recovery of LVDP. Chronologic changes in postischemic recovery of LVDP are shown in Fig 2. Very different recovery responses were obtained; in groups 3 and 5 to 9 , hearts recovered rapidly, reaching a plateau by 10 minutes of reperfusion (at this time point, these values were significantly higher than those of groups 1 , 2 , and 4). In contrast, groups 1,2 , and 4 recovered 


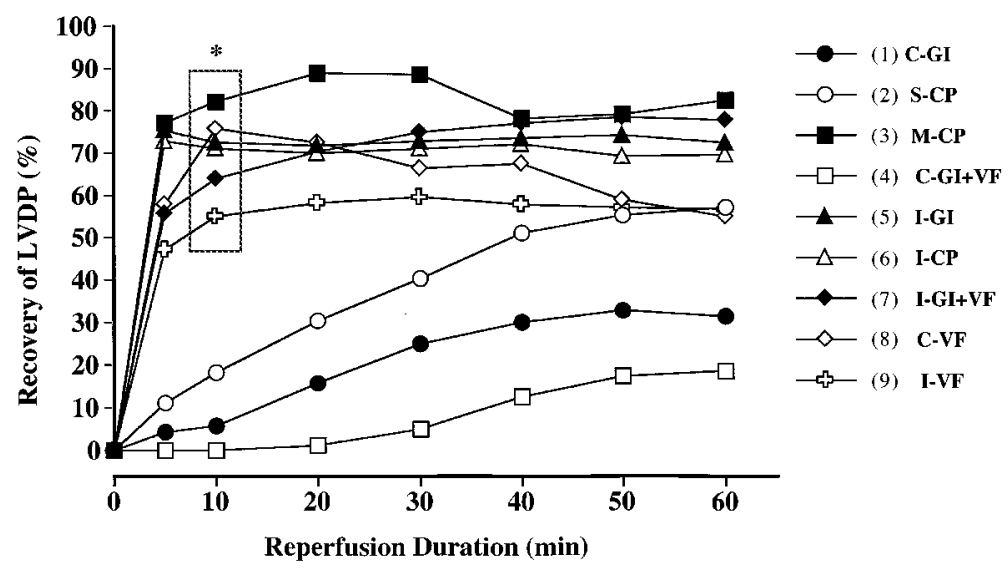

Fig 2. Recovery of LVDP with reperfusion duration (minutes), expressed as a percentage of the preischemic control value. ${ }^{*} P<.01$ : groups $3,5,6,7,8$, and 9 versus groups 1,2 , and 4 . Values are the mean of at least 6 hearts per group; error bars have been removed to improve clarity. $L V D P$, Left ventricular developed pressure; $C$ - $G I$, continuous global ischemia group; $S$ - $C P$, single-dose cardioplegic infusion group; $M-C P$, multidose cardioplegic infusion group; $C$ $G I+V F$, continuous global ischemia with ventricular fibrillation group; $I-G I$, intermittent global ischemia group; I-CP, intermittent cardioplegic infusion group; $I-G I+V F$, intermittent global ischemia with ventricular fibrillation group; $C$ $V F$, continuous ventricular fibrillation group; $I-V F$, intermittent ventricular fibrillation group.

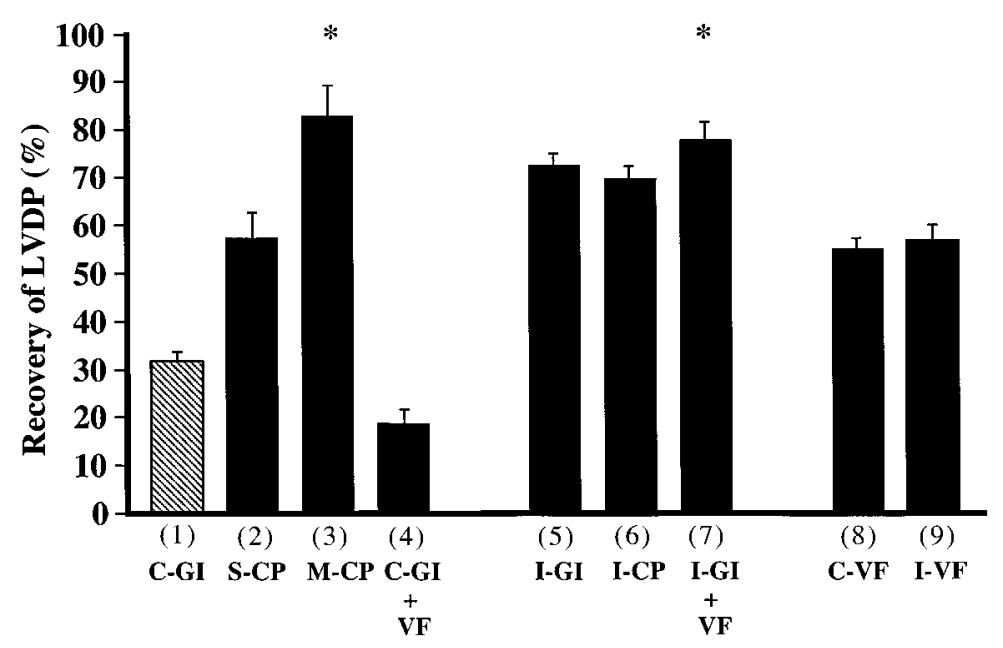

Fig 3. Recovery of LVDP at the end of 60 minutes of reperfusion, expressed as percentage of the preischemic control value. Values are the mean of at least 6 hearts per group \pm SEM. ${ }^{*} P<.05$ vs groups $1,2,4,8$, and $9 . L V D P$, Left ventricular developed pressure; $C$ - $G I$, continuous global ischemia group; $S$ - $C P$, single-dose cardioplegic infusion group; $M-C P$, multidose cardioplegic infusion group; $C-G I+V F$, continuous global ischemia with ventricular fibrillation group; $I-G I$, intermittent global ischemia group; $I-C P$, intermittent cardioplegic infusion group; $I$ $G I+V F$, intermittent global ischemia with ventricular fibrillation group; $C-V F$, continuous ventricular fibrillation group; $I-V F$, intermittent ventricular fibrillation group.

slowly and did not reach a plateau until 50 to 60 minutes of reperfusion. Recovery was poorest in hearts subjected to continuous (40 minutes) global ischemia (group 1) or continuous (40 minutes) global ischemia with VF (group 4); surprisingly, however, hearts pro- tected with a single infusion of STH2 before 40 minutes of continuous ischemia (group 2) also recovered relatively slowly.

The final recovery of LVDP after 60 minutes of reperfusion in all groups is shown in Fig 3 . The highest 
Table I. Baseline values (measured after 20 minutes of aerobic perfusion to achieve equilibration) of LVDP, coronary flow, heart rate, and LVEDP

\begin{tabular}{lrrrc}
\hline & \multicolumn{4}{c}{ Groups } \\
\cline { 2 - 5 } & $1: C$-GI & 2: $S$-CP & 3: M-CP & 4:C-GI+VF \\
\hline LVDP (mm Hg) & $110.8 \pm 4.1$ & $109.7 \pm 3.5$ & $119.3 \pm 5.6$ & $107.8 \pm 5.8$ \\
Coronary flow (mL/min) & $11.7 \pm 0.7$ & $10.6 \pm 0.9$ & $9.9 \pm 0.7$ & $9.5 \pm 0.7$ \\
Heart rate (beats/min) & $262.3 \pm 9.6$ & $274.2 \pm 12.5$ & $278.9 \pm 8.9$ & $290.8 \pm 13.3$ \\
LVEDP (mm Hg) & $5.8 \pm 0.6$ & $5.2 \pm 0.7$ & $5.5 \pm 0.6$ & $4.2 \pm 0.7$ \\
\hline
\end{tabular}

Values are mean \pm SEM. There were no differences between any of these groups. $L V D P$, Left ventricular developed pressure; $L V E D P$, left ventricular end-diastolic pressure; $C$ - $G I$, continuous global ischemia group; $S$ - $C P$, single-dose cardioplegic infusion group; $M$ - $C P$, multidose cardioplegic infusion group; $C$ - $G I+V F$, continuous global ischemia with ventricular fibrillation group; $I-G I$, intermittent global ischemia group; $I-C P$, intermittent cardioplegic infusion group; $I-G I+V F$, intermittent global ischemia with ventricular fibrillation group; $C-V F$, continuous ventricular fibrillation group; $I-V F$, intermittent ventricular fibrillation group.

Table II. Results of post hoc analysis for recovery of LVDP after 60 minutes of reperfusion

\begin{tabular}{lcccc}
\hline & & & P values & \\
\cline { 2 - 5 } & Group 1 & Group 2 & Group 3 & Group 4 \\
\hline Group 1: C-GI & - & $<.0001$ & $<.0001$ & .0288 \\
Group 2: S-CP & $<.0001$ & - & $<.0001$ & $<.0001$ \\
Group 3: M-CP & $<.0001$ & $<.0001$ & - & $<.0001$ \\
Group 4: C-GI+VF & .0288 & $<.0001$ & .0001 & $<-$ \\
Group 5: I-GI & $<.0001$ & .0074 & .0934 & $<.0001$ \\
Group 6: I-CP & $<.0001$ & .0271 & .4197 & $<.0001$ \\
Group 7: I-GI+VF & $<.0001$ & .0004 & $<.0001$ & $<.0001$ \\
Group 8: C-VF & .0009 & .6719 & $<.0001$ & $<.0001$ \\
Group 9: I-VF & .0005 & .9188 &
\end{tabular}

P value, Probability value; $L V D P$, left ventricular developed pressure; $C$ - $G I$, continuous global ischemia group; $S$ - $C P$, single-dose cardioplegic infusion group; $M$ - $C P$, multidose cardioplegic infusion group; $C-G I+V F$, continuous global ischemia with ventricular fibrillation group; $I-G I$, intermittent global ischemia group; $I-C P$, intermittent cardioplegic infusion group; $I-G I+V F$, intermittent global ischemia with ventricular fibrillation group; $C-V F$, continuous ventricular fibrillation group; $I-V F$, intermittent ventricular fibrillation group.

Table III. Characteristics of ischemic contracture (time to onset, time to peak, and peak contracture) in those groups in which a complete contracture occurred

\begin{tabular}{lcccc}
\hline & \multicolumn{3}{c}{ Groups } \\
\cline { 2 - 5 } & $1: C$ - $G I$ & $2: S$ - $C P$ & $3: M$-CP & 4: C-GI+VF \\
\hline Time to onset (min) & $15.3 \pm 1.1$ & $26.8 \pm 0.8^{*}$ & - & $11.4 \pm 0.3^{\dagger}$ \\
Time to peak (min) & $19.1 \pm 1.2$ & $30.5 \pm 0.9^{*}$ & - & $14.0 \pm 0.2^{\ddagger}$ \\
Peak (mm Hg) & $67.7 \pm 5.9$ & $50.0 \pm 2.9^{*}$ & - & $95.2 \pm 5.8^{\dagger}$ \\
\hline
\end{tabular}

There was either late or no contracture in groups 3 and 6. There was no contracture in groups 8 and 9, although LVEDP was elevated to around 40 mm Hg (see text). Values are mean \pm SEM. Abbreviations are as in Table I.

${ }^{*} P<.05$ versus group 1 .

${ }^{\dagger} P<.05$ versus groups 1,2 , and 5 .

${ }^{\ddagger} P<.05$ versus all groups.

$\S_{P}<.05$ versus group 5 .

degree of recovery was obtained in hearts subjected to multidose cardioplegic infusion (every 10 minutes) throughout 40 minutes of continuous global ischemia (group 3); however, similar recovery was obtained in hearts subjected to intermittent global ischemia with
VF (group 7). Interestingly, hearts from both these groups recovered to a significantly higher level than hearts protected with only a single infusion of cardioplegic solution and, as seen in Fig 2, the rate of recovery was also significantly more rapid. When compared 


\begin{tabular}{ccccc}
\hline \multicolumn{4}{c}{ Groups } \\
\hline 5: $I$ - $G I$ & 6: $I$-CP & 7: I-GI+VF & 8: C-VF & 9: I-VF \\
\hline $111.8 \pm 6.2$ & $127.7 \pm 3.3$ & $111.8 \pm 5.5$ & $122.7 \pm 4.7$ & $111.5 \pm 6.7$ \\
$10.5 \pm 0.7$ & $10.4 \pm 0.6$ & $11.2 \pm 1.4$ & $11.8 \pm 0.5$ & $11.2 \pm 0.7$ \\
$269.1 \pm 13.3$ & $262.1 \pm 14.4$ & $284.2 \pm 15.0$ & $292.0 \pm 13.7$ & $283.1 \pm 11.3$ \\
$4.5 \pm 0.3$ & $5.3 \pm 1.0$ & $5.3 \pm 0.5$ & $4.3 \pm 0.4$ & $5.7 \pm 0.6$ \\
\hline
\end{tabular}

\begin{tabular}{|c|c|c|c|c|}
\hline \multicolumn{5}{|c|}{ P value } \\
\hline Group 5 & Group 6 & Group 7 & Group 8 & Group 9 \\
\hline$<.0001$ & $<.0001$ & $<.0001$ & .0009 & .0005 \\
\hline .0074 & .0271 & .0004 & .6719 & .9188 \\
\hline .0934 & .034 & .4197 & $<.0001$ & $<.0001$ \\
\hline$<.0001$ & $<.0001$ & $<.0001$ & $<.0001$ & $<.0001$ \\
\hline- & .6414 & .3743 & .005 & .011 \\
\hline .6414 & - & .1783 & .0171 & .034 \\
\hline .3743 & .1783 & - & .0004 & .0009 \\
\hline .005 & .0171 & .0004 & - & .7689 \\
\hline .011 & .034 & .0009 & .7689 & - \\
\hline
\end{tabular}

\begin{tabular}{ccccc}
\hline & Groups & & \\
\hline $5: I-G I$ & $6: I-C P$ & $7: I-G I+V F$ & 8: $C$-VF & 9: I-VF \\
\hline $18.6 \pm 1.4$ & - & $14.0 \pm 1.1^{\S}$ & - & - \\
$36.2 \pm 3.4$ & - & $20.2 \pm 1.9^{\S}$ & - & - \\
$66.0 \pm 4.6$ & - & $89.3 \pm 5.3^{\S}$ & - & - \\
\hline
\end{tabular}

with hearts subjected to continuous (40 minutes) global ischemia (group 1; control), all but one group had a significantly better degree of recovery; the exception was group 4, hearts in which VF was imposed on global ischemia, which showed a tendency for a lower degree of recovery (although this difference did not reach significance). The significance of between-group post hoc analysis for LVDP is shown in Table II.
Changes in LVEDP with time. Ischemic contracture (elevated LVEDP) occurred in all groups subjected to global ischemia (groups 1-7); Table III shows time to onset (minutes), time to peak (minutes), and peak (millimeters of mercury) of contracture. In hearts from groups 3 and 6, either no contracture or late and incomplete contracture occurred; consequently, contracture measurements were not 
possible in these hearts. In addition, hearts subjected to VF with maintained perfusion (groups 8 and 9) did not exhibit contracture, although LVEDP was increased to around $40 \mathrm{~mm} \mathrm{Hg}$ (range $34-56 \mathrm{~mm} \mathrm{Hg}$ ) as a result of the VF. As shown in Table III, similar contracture measurements were obtained in hearts subjected to global ischemia alone (either continuous or intermittent), but the degree of recovery of LVDP was significantly higher with intermittent ischemia (Fig 3). Cardioplegic protection, however, prolonged the ischemic duration before contracture occurred and the extent was reduced, although the recovery of LVDP was similar to that of intermittent ischemia. In contrast, VF associated with either continuous or intermittent global ischemia significantly shortened the ischemic duration before the occurrence of contracture and significantly increased the extent of contracture. Although the contracture characteristics with VF were very similar, the extent of recovery of LVDP was different; recovery after continuous ischemia and VF was poor whereas it was good after intermittent ischemia and VF.

Changes in postischemic LVEDP with time during reperfusion are shown in Fig 4. At the onset of reperfusion, LVEDPs in all groups were relatively high (varying between 35.2 and $58.7 \mathrm{~mm} \mathrm{Hg}$ ); however, in groups 1, 2, and 4, LVEDP increased further to a peak at 5 minutes, subsequently reducing during the rest of the reperfusion period but still remaining significantly elevated. In all other groups, LVEDP was not different from baseline values and there were no differences in between-group values.

\section{Discussion}

The present study demonstrated that, with the same cumulative duration of ischemia, intermittent crossclamping with VF provided a similar level of myocardial protection as that achieved with multidose cardioplegic ischemic arrest. In addition, myocardial protection by intermittent crossclamping with VF was more effective than single-dose cardioplegic ischemic arrest and either continuous or intermittent VF with coronary perfusion.

In the 1970s, numerous experimental studies into the myocardial protective effect of VF were undertaken. ${ }^{12-16}$ Studies from Buckberg's group ${ }^{12,15}$ demonstrated that electrically maintained VF caused subendocardial ischemia, shown by acidosis, potassium loss, and a fall in lactate extraction, all of which contributed to subendocardial injury and impaired post-VF function despite apparently normal coronary perfusion. In further studies, Hottenrott and Buckberg ${ }^{13}$ presented evidence that ventricular distention secondary to VF added to the occurrence of subendocardial underperfusion. The suggested mechanism by which flow was shunted away from the subendocardium during VF was the compressive force exerted on subendocardial vessels, either by the strength of VF or from elevated intracavity pressure caused by malfunction of the ventricular vent, leading to the evolution of myocardial edema as ischemia was prolonged. ${ }^{14}$ Moreover, Hearse, Stewart, and Chain ${ }^{16}$ demonstrated that, in hearts subjected to electrically induced VF during coronary perfusion, the recovery of cardiac function was reduced and myocardial adenosine triphosphate and creatine phosphate levels were considerably depleted, indicating increased myocardial energy consumption.

In our study, intraventricular pressure (LVEDP) increased in hearts subjected to VF with coronary perfusion (groups 8 and 9) to approximately $40 \mathrm{~mm} \mathrm{Hg}$ (range $34-56 \mathrm{~mm} \mathrm{Hg}$ ). In these hearts, coronary flow was maintained close to (or higher than) baseline values, which suggests that adequate coronary perfusion was maintained. From our data, however, it was not possible to determine whether underperfusion occurred in the subendocardium ${ }^{13}$; it is possible that the maintained coronary flow reflects an increased flow in the other regions (coronary steal). This theory may be supported by our observation that continuous or intermittent VF, in combination with continuous perfusion during VF (groups 8 and 9, respectively), produced a significant reduction in recovery of function when compared with either multidose cardioplegia or intermittent crossclamping with VF (groups 3 and 7, respectively).

The role of left ventricular distention on myocardial injury has been evaluated in isolated cat hearts perfused with crystalloid solution and subjected to cardioplegic ischemic arrest or VF with maintained coronary perfusion. ${ }^{17}$ Increased LVEDP (induced by inflating an intraventricular balloon) during ischemia did not influence myocardial gas tension, left ventricular function, coronary blood flow, endocardial/epicardial blood flow ratio, or myocardial water content. In contrast, fibrillating hearts with maintained perfusion exhibited decreased function, impaired subendocardial blood flow, and elevated carbon dioxide tension, confirming previous results $^{12-15}$ of the detrimental effects of VF. Thus, left ventricular distention does not appear to be detrimental during periods of ischemia (no coronary flow), but distention with VF and maintained coronary perfusion is harmful. Our data support this theory; hearts in groups 8 and 9, which were subjected to VF with maintained perfusion (in which LVEDP was around $40 \mathrm{~mm} \mathrm{Hg}$ ) recovered to a significantly lesser degree than hearts subjected to intermittent ischemia with $\mathrm{VF}$ (group 7), in which contracture-generated LVEDPs rose to around $90 \mathrm{~mm} \mathrm{Hg}$. In 


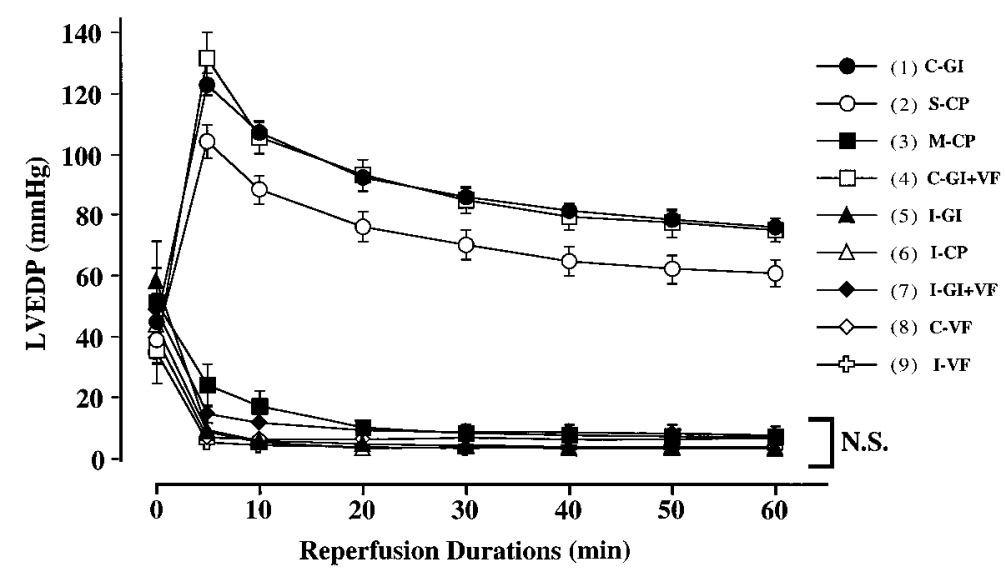

Fig 4. Changes in LVEDP during reperfusion (expressed as absolute value in millimeters of mercury). Values are the mean of at least 6 hearts per group \pm SEM. LVEDP, Left ventricular end-diastolic pressure; N.S., nonsignificant; $C$-GI, continuous global ischemia group; $S$ - $C P$, single-dose cardioplegic infusion group; $M$ - $C P$, multidose cardioplegic infusion group; $C-G I+V F$, continuous global ischemia with ventricular fibrillation group; $I-G I$, intermittent global ischemia group; $I-C P$, intermittent cardioplegic infusion group; $I-G I+V F$, intermittent global ischemia with ventricular fibrillation group; $C-V F$, continuous ventricular fibrillation group; $I-V F$, intermittent ventricular fibrillation group.

addition, our results indicate that application of VF during crossclamp ischemia (group 7), which is applied to induce a still, nonbeating heart during surgery, does not exacerbate any ischemic injury (group 5). These results confirm the relative safety of using intermittent crossclamping with VF during coronary artery bypass surgery.

In this study, the lowest degree of recovery of LVDP was observed in hearts in which VF was induced throughout continuous (40 minutes) global ischemia (group 4); in contrast, intermittent global ischemia with the same total cumulative ischemic duration (40 minutes), either with or without induction of VF (groups 7 and 5, respectively), recovered to a significantly higher level. Similar results have previously been shown by Engelman and colleagues ${ }^{18}$ in nonhypertrophic pig hearts. When normothermic ischemic arrest with VF was induced for 15 minutes, no maldistribution of coronary flow or loss of the hyperemic response was observed, even if this protocol was repeated 6 times with only 5 minutes of reperfusion separating the ischemic intervals each time. In contrast, prolonging the duration of ischemia with VF to 30 minutes (again with only 5 minutes of reperfusion between intervals) led to a maldistribution of flow away from the endocardium, as well as the loss of the hyperemic response. Thus, the addition of VF to ischemia had no significant detrimental effect when the ischemic durations were short (even up to 15 minutes) but, with prolonged ischemia and VF, severe myocardial damage occurred.
Our results showed that intermittent global ischemia (with or without VF) preserved postischemic recovery of LVDP to a level similar to that of multidose cardioplegia. It is tempting to speculate that intermittent ischemia may be protecting the myocardium in a manner similar to that of ischemic preconditioning ${ }^{19}$; however, there are a number of differences between intermittent ischemia and classic preconditioning. The ischemic period is longer than those usually used to induce a preconditioning protection, although some studies $^{20}$ have used similar durations (2 episodes of 8 minutes of ischemia and 8 minutes of reperfusion) and obtained excellent preconditioning protection in pig hearts. The most obvious difference is the absence of the prolonged ischemic period after the preconditioning stimulus, but it is possible that the initial ischemiareperfusion episode protects the subsequent episodes. In fact, this was the question addressed in a study of cardiopulmonary bypass in dogs by Abd-Elfattah, Ding, and Wechsler ${ }^{21}$; they used a protocol similar to that of the present study, comparing 60 minutes of normothermic global ischemia to 6 episodes of 10 minutes of normothermic global ischemia and 10 minutes of reperfusion. Interestingly, adenosine triphosphate levels were maintained after the second episode of ischemia and reperfusion and were significantly higher at the end of the intermittent protocol than after sustained ischemia. In addition, purine release was limited and cardiac function was significantly better than that obtained with the sustained ischemia group. It was sug- 
gested that the intermittent ischemia and reperfusion augmented the endogenous protective mechanism or mechanisms of "preconditioning." However, the present study was not intended to examine whether intermittent global ischemia (either with or without VF) exerted any protective effect by a preconditioning mechanism, although further studies appear warranted.

Marked differences exist between the present study, the more recent clinical studies, ${ }^{5-11}$ and previous experimental and clinical investigations, ${ }^{22-25}$ in which a loss of cardiac protection was demonstrated when intermittent crossclamping (global ischemia) was used. These differences involve the relative durations of ischemia and reperfusion that were used; when repeated episodes of ischemia of around 10 to 20 minutes in duration were interspersed with reperfusion durations of only 3 to 5 minutes, poor cardiac protection was observed. Thus, in these studies, the ischemic duration was 3 to 6 times longer than the reperfusion interval. In contrast, in those studies in which a cardioprotective effect of intermittent crossclamping has been demonstrated, ${ }^{5-11,26}$ a protocol was used in which repeated episodes of 10 minutes of ischemia were followed by 10 to 15 minutes of reperfusion; thus, the reperfusion duration was at least as long as the preceding duration. These results were similar to those achieved in the present study, in which intermittent ischemic durations of 10 minutes, followed by reperfusion for 10 minutes, resulted in a high level of recovery of function, regardless of whether VF was induced during ischemia. It has previously been shown ${ }^{27}$ in the pig heart that myocardial injury associated with intermittent ischemia and reperfusion can be exacerbated by coincident VF, by shortening the period of reperfusion, extending the periods of ischemia, or by using high perfusion pressure. Of these factors, the most critical determinant to sustain adequate subendocardial perfusion was the duration of reperfusion. Benzing and coworkers $^{22}$ concluded that the longer the individual ischemic intervals or the shorter the periods of reperfusion, the greater is the likelihood of tissue injury. Thus, the time ratio of ischemic duration to reperfusion duration appears to be the important factor in determining how well the myocardium will recover. Ischemic preconditioning is induced by a short period (2-5 minutes) of ischemia followed by a period of reperfusion (5-10 minutes) before the sustained period of ischemia. A growing body of evidence suggests that the duration and number of cycles of ischemia and reperfusion necessary to confer protection may vary between species and between the end points assessed. ${ }^{28}$ In studies in which the time ratio of ischemia to reperfusion is high (such as was the case in previous studies ${ }^{22-25}$ ), any potential benefit that might have occurred as a result of ischemic preconditioning was not seen. In fact, this high ratio, together with the longer total ischemic time, caused severe myocardial injury.

In fairness to these earlier studies, the protocols of ischemia and reperfusion used in basic experimental studies were influenced by the clinical techniques of myocardial protection at that time. In the 1970s, intermittent crossclamping was used not only during coronary artery bypass surgery but also during other types of cardiac operations, such as valve replacement for valve disease or intracardiac repair for congenital heart disease. During cardiac operations, it is not possible to continue the operation during reperfusion periods; consequently, the duration of ischemia had to be much longer than the duration of reperfusion. Coronary artery bypass surgery, however, is the exception because the proximal ends of grafts can be sutured during reperfusion. Considering the circumstances mentioned above, we would suggest that intermittent ischemia with VF is suitable for myocardial preservation during coronary artery surgery but is not a good technique to protect the myocardium during other cardiac operations.

\section{Limitations of the study}

We concede that the present findings were made in the normal rat heart. Therefore, we could not evaluate the effect of cardioplegic maldistribution and any potential disadvantage (or advantage) of intermittent crossclamping with VF in the diseased heart. ${ }^{14,21}$ Another possible limitation of the present study may be the use of an isolated heart preparation that does not possess a collateral circulation (a notable difference from the clinical situation). In these studies, VF was maintained by continuous alternating current stimulation. This was necessary because the rat heart is too small (has insufficient mass of ventricular tissue) for VF to be maintained from a short stimulus (the "critical mass" theory). We appreciate that either a short VF stimulus or spontaneous VF is sufficient for $\mathrm{VF}$ to be maintained in the clinical setting and that these differences may influence the outcome of the results, since spontaneous VF has been shown to be less damaging. ${ }^{12}$

\section{Conclusion}

Optimal myocardial protection was obtained both with multidose crystalloid cardioplegia during continuous global ischemia and with intermittent crossclamping (either with or without VF). This study demon- 
strates that intrinsic myocardial protection was obtained with intermittent crossclamping with VF, and therefore this technique should provide good myocardial protection during coronary artery bypass surgery. Further studies are warranted into the mechanism by which this protection is achieved and into the optimal ratio of ischemia to reperfusion duration.

\section{REFERENCES}

1. Izzat MB, West RR, Bryan AJ, Angelini GD. Coronary artery bypass surgery: current practice in the United Kingdom. Br Heart J 1994;71:382-5.

2. Robinson LA, Schwarz GD, Goddard DB, Fleming WH, Galbraith TA. Myocardial protection for acquired heart disease surgery: results of a national survey. Ann Thorac Surg 1995;59:361-72.

3. Akins CW, Carroll DL. Event-free survival following nonemergency myocardial revascularization during hypothermic fibrillatory arrest. Ann Thorac Surg 1987;43:628-33. Updated in 1994: Akins CW. Early and late results following emergency isolated myocardial revascularization during hypothermic fibrillatory arrest. Ann Thorac Surg 1994;58:1205-6.

4. Bonchek LI, Burlingame MW, Vazales BE, Lundy EF, Gassmann CJ. Applicability of noncardioplegic coronary bypass to high-risk patients: selection of patients, technique, and clinical experience in 3000 patients. J Thorac Cardiovasc Surg 1992;103:230-7.

5. Gerola LR, Oliveira SA, Moreira LFP, Dallan LAO, da Luz PL, Jatene AD. Blood cardioplegia with warm reperfusion versus intermittent aortic crossclamping in myocardial revascularization: randomized controlled trial. J Thorac Cardiovasc Surg 1993;106:491-6.

6. Taggart DP, Bhusari S, Hooper J, Kemp M, Magee P, Wright JE, et al. Intermittent ischaemic arrest and cardioplegia in coronary artery surgery: coming full circle? Br Heart J 1994;72:136-9.

7. Anderson JR, Hossein-Nia M, Kallis P, Pye M, Holt DW, Murday $\mathrm{AJ}$, et al. Comparison of two strategies for myocardial management during coronary artery operations. Ann Thorac Surg 1994;58:768-73.

8. Alhan HC, Karabulut H, Tosun R, Karakoc F, Okar I, Demiray E, et al. Intermittent aortic cross-clamping and cold crystalloid cardioplegia for low-risk coronary patients. Ann Thorac Surg 1996;61:834-9.

9. Cohen AS, Hadjinikolaou L, McColl A, Richmond W, Sapsford RA, Glenville BE. Lipid peroxidation, antioxidant status and troponin- $\mathrm{T}$ following cardiopulmonary bypass: a comparison between intermittent crossclamp with fibrillation and crystalloid cardioplegia. Eur J Cardiothorac Surg 1997;12:248-53.

10. Liu Z, Valencia O, Treasure T, Murday JA. Cold blood cardioplegia or intermittent cross-clamping in coronary artery bypass grafting? Ann Thorac Surg 1998;66:462-5.

11. Musumeci F, Feccia M, MacCarthy PA, Ellis GR, Mammana L, Brinn F, et al. Prospective randomised trial of single clamp technique versus intermittent ischaemic arrest: myocardial and neurological outcome. Eur J Cardiothorac Surg 1998;13:702-9.

12. Hottenrott CE, Maloney JV, Buckberg GD. Studies of the effects of ventricular fibrillation on the adequacy of regional myocardial flow. I. Electrical versus spontaneous fibrillation. J Thorac Cardiovasc Surg 1974;68:615-25.
13. Hottenrott CE, Buckberg GD. Studies of the effects of ventricular fibrillation on the adequacy of regional myocardial flow. II. Effects of ventricular distention. J Thorac Cardiovasc Surg 1974;68:626-33

14. Hottenrott CE, Maloney JV, Buckberg GD. Studies of the effects of ventricular fibrillation on the adequacy of regional myocardial flow. III. Mechanisms of ischemia. J Thorac Cardiovasc Surg 1974;68:634-45

15. Buckberg GD, Hottenrott CE. Ventricular fibrillation: its effect on myocardial flow, distribution, and performance. Ann Thorac Surg 1975;20:76-85.

16. Hearse DJ, Stewart DA, Chain EB. Recovery from cardiac bypass and elective cardiac arrest: the metabolic consequences of various cardioplegic procedures in the isolated rat heart. Circ Res 1974;35:448-57.

17. Lucas SK, Gardner TJ, Elmer EB, Flaherty JT, Bulkley BH, Gott VL. Comparison of the effects of left ventricular distention during cardioplegic-induced ischemic arrest and ventricular fibrillation. Circulation 1980;62:142-9.

18. Engelman RM, Adler S, Gouge TH, Chandra R, Boyd AD, Bauman FG. The effect of normothermic anoxic arrest and ventricular fibrillation on the coronary blood flow distribution of the pig. J Thorac Cardiovasc Surg 1975;69:858-69.

19. Murry CE, Jennings RB, Reimer KA. Preconditioning with ischemia: a delay of lethal cell injury in ischemic myocardium. Circulation 1986;74:1124-36.

20. Shattock MJ, Lawson CS, Hearse DJ, Downey JM. Electrophysiological characteristics of repetitive ischemic preconditioning in the pig heart. J Mol Cell Cardiol 1996;28:133947.

21. Abd-Elfattah AS, Ding M, Wechsler AS. Intermittent aortic crossclamping prevents cumulative adenosine triphosphate depletion, ventricular fibrillation, and dysfunction (stunning): Is it preconditioning? J Thorac Cardiovasc Surg 1995;110:328-39.

22. Benzing G, Stockert J, Nave E, Kaplan S. Intermittent myocardial ischemia during cardiopulmonary bypass. J Thorac Cardiovasc Surg 1973;65:108-11.

23. Brown AH, Braimbridge MV, Darracott S, Chayen J, Kasap H. An experimental evaluation of continuous normothermic, intermittent hypothermic, and intermittent normothermic coronary perfusion. Thorax 1974;29:38-50.

24. Follette MD, Mulder DG, Maloney JV, Buckberg GD. Advantages of blood cardioplegia over continuous coronary perfusion or intermittent ischemia: experimental and clinical study. J Thorac Cardiovasc Surg 1978;76:604-19.

25. Roberts AJ, Abel RM, Alonso DR, Subramanian VA, Paul JS, Gay WA Jr. Advantages of hypothermic potassium cardioplegia and superiority of continuous versus intermittent aortic crossclamping. J Thorac Cardiovasc Surg 1980;79:44-58.

26. Van der Veen FH, Van der Vusse GJ, Willemsen P, Kruger RT, Van der Nagel T, Coumans WA, et al. Changes in myocardial high-energy phosphate stores and carbohydrate metabolism during intermittent aortic crossclamping in dogs on cardiopulmonary bypass at $34^{\circ} \mathrm{C}$ and $25^{\circ} \mathrm{C}$. J Thorac Cardiovasc Surg 1990;100:389-99.

27. Engelman RM, Levitsky S, Wyndham CRC. Optimal condition for reperfusion during cardiopulmonary bypass. Circulation 1977;56(Suppl):II-148-59.

28. Cave AC. Preconditioning induced protection against postischemic contractile dysfunction: characteristics and mechanisms. J Mol Cell Cardiol 1995;27:969-79. 